\title{
Detección de fallas de arco eléctrico en redes eléctricas domesticas AC basado en el análisis de la tensión y corriente
}

\author{
${ }^{1}$ Jinmi Lezama ${ }^{2}$ Patrick Schweitzer, ${ }^{2}$ Serge Weber \\ ${ }^{1}$ Universidad Nacional Tecnológica de Lima Sur, Lima Perú. \\ ${ }^{2}$ Universidad de Lorraine, Lorraine - Francia.
}

Recibido: 15/04/2021 Revisado: 21/05/2021 Aceptado: 26/06/2021 Publicado: 28/07/2021

\section{Resumen}

La protección de las instalaciones eléctricas basadas en disyuntores electromagnéticos permite la detección de fallas de tipo cortocircuito y sobre corriente, sin embargo, se ha observado la presencia de fallas de tipo arco eléctrico, los cuales han sido los iniciadores de incendios en viviendas de Estados Unidos y Europa, debido a que las fallas de tipo arco eléctrico serie pueden generar altas temperaturas. Este articulo presenta un método de detección de fallas de arco eléctrico serie basado en el análisis de la forma de onda y la rapidez de cambio del flujo de corriente al momento de la producción de la descarga entre dos electrodos. Los resultados presentados muestran la posibilidad del uso de este método para la detección de fallas eléctricas y fallas de tipo arco eléctrico serie en las instalaciones eléctricas.

Palabras clave: Incendios, arco eléctrico serie, disyuntores, análisis de señales, algoritmos

\begin{abstract}
Protection of electrical installations based on electromagnetic circuit breakers allows the detection of short- circuit and overcurrent faults, however, the presence of arcing faults has been observed, which have been the initiators of fires in homes in the United States and Europe, because arcing faults can generate high temperatures. This paper presents a method for detecting series arcing faults based on the analysis of the signature and the rate of change of current, when the restrike voltage is reached between two electrodes. The results presented show the possibility of using this method for the detection of electrical faults and series arc faults in electrical installations.
\end{abstract}

Keywords: Fire, serial arc fault, circuit breakers, signal analysis, algorithms 


\section{Introducción}

Las redes de distribución y transmisión eléctrica AC y DC pueden presentar diferentes tipos de fallas como son: corto circuito, fallas por sobre carga, fallas de arcos eléctricos, etc. que podrían generar un mal funcionamiento de los equipos eléctricos y/o también ser la fuente de la iniciación de diferentes incidentes (incendios, electrocución, etc...). Las fallas de origen eléctrico son una de las principales causas de incendios en viviendas en Estados Unidos y Europa.

En el 2014, según el reporte de emergencias atendidas a nivel nacional por el cuerpo general de bomberos del Perú, indican que ha existido un total de 9430 incendios, de los cuales el 35\% fueron de origen eléctrico, y de estos 1703 casos son emergencias por cortocircuitos, de las cuales son atribuidas directamente a cortocircuitos y femémonos ligados a los cortocircuitos $\mathrm{u}$ otro tipo de fallas eléctricas.

En la actualidad los sistemas eléctricos de baja tensión AC cuentan con dispositivos de protección denominados interruptores magnetotérmicos capaces de proteger un circuito eléctrico contra fallos de corto circuito y sobre corriente basados en el análisis magnético y térmico de la corriente eléctrica cuando un fallo es producido. Sin embargo, estudios recientes han demostrado la existencia de otro tipo de fallas que podrían originar el riesgo de incendios en las instalaciones eléctricas, estas fallas son denominadas de tipo arco eléctrico, producidas por instalaciones defectuosas, conductores dañados, mala conexión de conductores, el envejecimiento de instalaciones, etc. Siendo un motivo importante detectar estos fallos para tratar de prevenir la producción de incendios. Una falla de arco eléctrico es producida por una interrupción galvánica de la fuente de alimentación, por lo cual el aire se ioniza ante descargas eléctricas, generando un puente conductor de corriente sin contacto, si el arco se mantiene durante un suficientemente largo el arco serie puede liberar una gran cantidad de energía, llegando a alcanzar una temperatura de $5000{ }^{\circ} \mathrm{C}$ (Floyd, 2003), generando la carbonización del cable y el inicio de un incendio. Si el material que se encuentra en contacto al arco eléctrico es inflamable, existe una gran posibilidad de generación del incendio. Debido a que un arco eléctrico es un fenómeno que se presenta con una impedancia característica, los valores de tensión y corriente no son elevados, por lo cual un disyuntor termomagnético tradicional no podrá detectar este tipo de fenómenos. 
La norma UL (UL1699) de los Estados Unidos impone el uso de dispositivos electromagnéticos que detecten fallas de origen eléctrico, denominados Arc Fault Circuit Interrupter (AFCI) (Underwriters Laboratories, 2008). En el caso europeo la norma IEC 62606 (IEC62606, 2013) impulsa el uso de dispositivos termomagnéticos de detección de fallas de origen eléctrico.

Diversas técnicas son propuestas para la detección de estas fallas, las cuales son basados principalmente en el análisis de frecuencias y de forma, tomando en consideración la gran variedad de cargas eléctricas domesticas presentes en una red AC que podrían influenciar sobre el método y generar una falsa detección.

Los distintos métodos están basados en el análisis frecuencial, como es el análisis del rango de frecuencias hasta $20 \mathrm{kHz}$, las variaciones del 3er y 5to armónico (Artale, 2017), las variaciones a alta frecuencia superior a $20 \mathrm{MHz}(\mathrm{Bao}, 2019)$ y evaluación de armónicos $(\mathrm{Qu}$, 2018) pares e impares. De la misma forma otros métodos están basados en el análisis en el dominio temporal (Yanq, 2020) como la variación armónica, la autocorrelación, la transformada de wavelet (Qi, P, 2017), redes neuronales (Le, 2020), entre otros.

El articulo presenta la evaluación de un algoritmo basado en el análisis del cambio rápido de conducción de corriente, mediante el cálculo de la derivada, para la detección de una falla de origen eléctrico en distintas configuraciones de posicionamiento de la carga.

\section{Metodología de trabajo}

El desarrollo del artículo es dividido en 4 fases; La primera fase es la adquisición de señales de corriente y tensión AC de sistemas en funcionamiento normal y con la presencia de una falla de arco eléctrico serie generada de forma experimental en laboratorio. La segunda fase se encuentra en el estudio y validación de la técnica de detección de fallas de arco eléctrico y en la tercera fase, se realizará las pruebas de evaluación del algoritmo para distintas configuraciones de carga.

\section{II.1. Generación de fallas eléctricas.}

La generación del arco eléctrico en régimen AC (según la figura 1), es producida por una descarga debido a la ionización del medio entre los 2 electrodos. Donde la corriente AC genera una alternancia del campo eléctrico entre el ánodo y cátodo provocando un desplazamiento alterno de los electrones, la frecuencia alternativa baja $\mathrm{AC}$ de 50 o $60 \mathrm{~Hz}$ permite que un electrón pueda alcanzar el ánodo facilmente.

BIOTECH \& ENGINEERING Untels. Jul - Dic.1(2), 2021; ISSN:2788 -4295; 41-51 


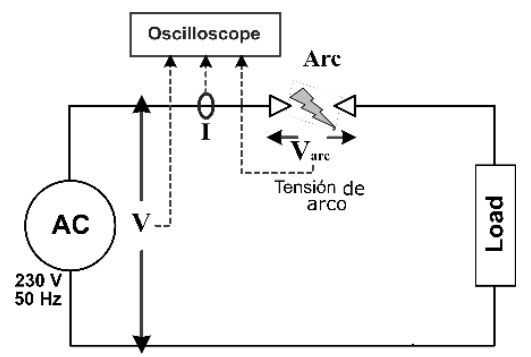

Figura 1. Circuito de generación de falla de arco eléctrico serie.

La figura 2 muestra la forma de onda de la corriente (azul), la tensión (rojo) y la tensión de arco (negro) cuando una falla de arco eléctrico serie es producido. En esta figura se observa que, al momento del paso por cero de la corriente, esta se mantiene hasta que la tensión entre los dos electrodos pueda alcanzar la tensión de restrike, es en este momento que se produce la descarga eléctrica y el flujo de electrones (la corriente eléctrica que está en razón de la tensión de alimentación y la carga) hasta el siguiente paso por cero.

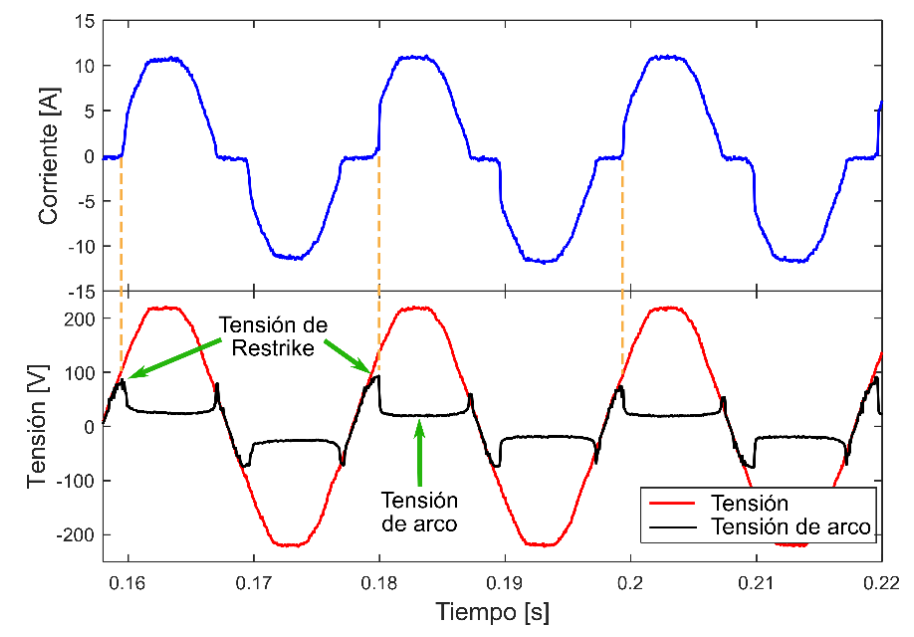

Figura 2. Forma de onda de corriente y tensión de arco de una carga resistiva

\section{II.2. Test de generación de fallas de arco eléctrico en distintas cargas}

La forma de onda de una falla de arco eléctrico puede variar en función de la carga eléctrica conectada en el circuito, en este articulo analizaremos la forma de onda de la corriente para una carga de tipo resistivo (figura 3), una carga de tipo motor (figura 4), una carga de tipo variador de intensidad (figura 5) y una carga de tipo escondida que consta de una resistencia en paralelo con una falla de arco eléctrico y un variador de intensidad (figura 6).

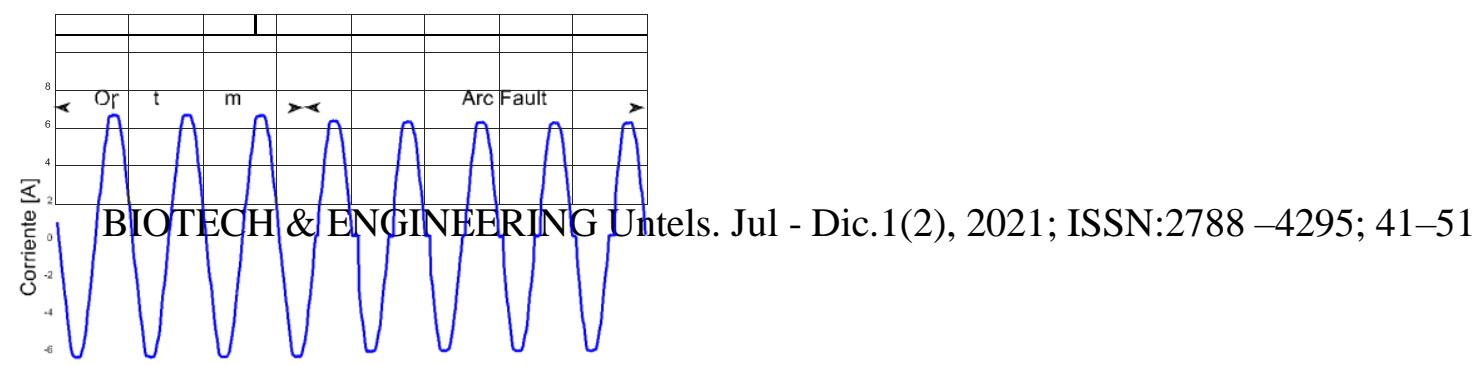




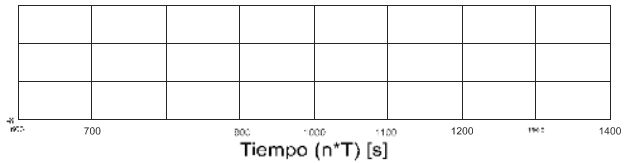

Figura 3. Carga resistiva

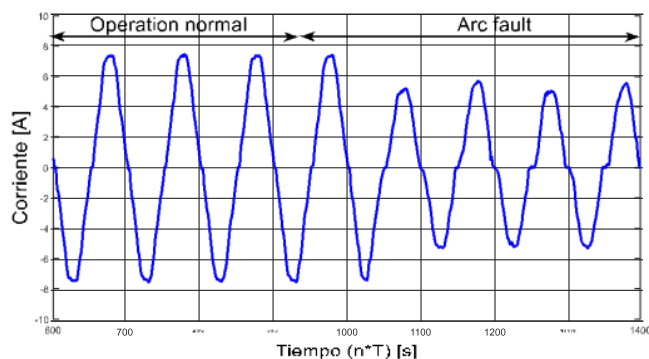

Figura 4. Carga tipo motor

\section{II.3. Métodos de detección basado en la derivada de la corriente.}

El algoritmo se basa en el análisis del cambio de régimen de una señal (Johnson, 2012) (Tisserand, 2015). La figura 7 muestra la base teórica del método que detecta una discontinuidad de la señal, observada a partir de una variación de nivel. Siendo el cálculo de la derivada definido como la razón de cambio entre 2 puntos de análisis, lo cual ser una variable que evidencia un cambio fiable al momento de la producción de un arco eléctrico.

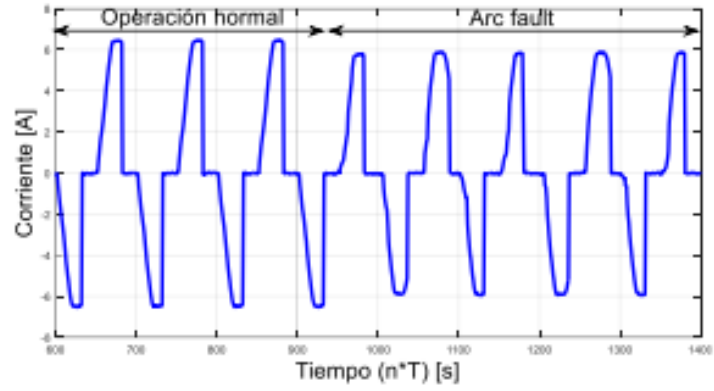

Figura 5. Carga variador de intensidad

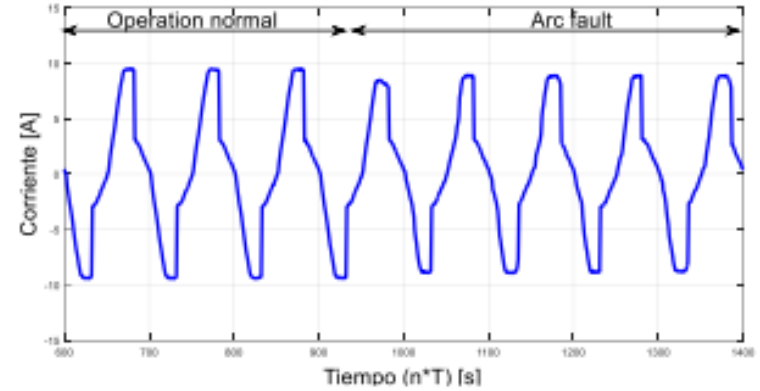

Figura 6. Carga escondida

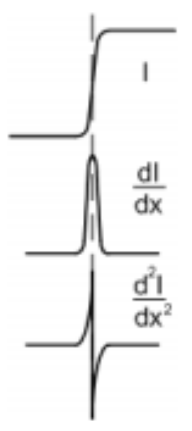

Figura 7. Detección de la discontinuidad en una señal

Si consideramos una función $\mathrm{y}(\mathrm{x})$ definida sobre un intervalo de $\mathrm{N}$ valores discretos $0<\mathrm{x}<\mathrm{N}$. La señal es muestreada periódicamente a una razón de $\mathrm{x}(\mathrm{k})=\mathrm{kT}$. La estimación de la primera derivada numérica de $\mathrm{y}(\mathrm{x})$ en el punto $\mathrm{x}$ es descrito según la ecuación 1. 


$$
y^{\prime}(x) \approx \frac{y\left(x_{1}\right)-y\left(x_{0}\right)}{x_{1}-x_{0}}, x \in\left[x_{0}, x_{1}\right]
$$

De donde la expresión de la derivada puede ser expresada en la ecuación 2, el cual es obtenido para cada una de las muestras adquiridas

$$
y^{\prime}\left(x_{k}\right)=\frac{y\left(x_{k}\right)-y\left(x_{k-1}\right)}{T}+\mathcal{O}(T)
$$

A partir de la ecuación 2 se extrae una segunda ecuación de diferencia centrado en el orden 2 para $y(x)$. De acuerdo a la ecuación 3, podemos observar que para una diferencia de orden 1 , el error vario linealmente a razón de $\mathrm{T}$ y para el caso de la diferencia de orden 2 la diferencia varía según $\mathrm{T}^{2}$. En este sentido para una función de "y" suficientemente liza y para un pequeño paso de T, la diferencia de orden 2 generara un error más pequeño. Por otro lado, un problema para tener en cuenta en este método es la sensibilidad al ruido de medida.

$$
y^{\prime}\left(x_{k}\right)=\frac{y\left(x_{k+1}\right)-y\left(x_{k-1}\right)}{2 T}+\mathcal{O}\left(T^{2}\right)
$$

\section{II.4. Principio de detección.}

El método de calculo de la derivada es basado sobre la detección del cambio rápido de corriente. Según la teoría, un arco eléctrico es producido cuando la tensión aplicada alcanza un nivel suficiente para iniciar una descarga entre dos electrodos (denominado tensión de restrike), la corriente eléctrica, al momento del restrike, comienza un cambio rápido, pasando de un nivel cero amperios de corriente a una zona de conducción proporcional a la relación de tensión y la impedancia (Figura 2).

Con el fin de incrementar la razón de cambio de la derivada de corriente, es posible realizar el cálculo de la tercera derivada de la corriente. La principal idea de realizar este cálculo es con el fin de obtener de razón fiable las transiciones rápidas de corriente cuando la producción de un arco es efectuada. La primera derivada expresa las variaciones dadas a un cambio de forma de onda, pero esta no es muy importante. De acuerdo a la ecuación 4, la obtención de la tercera derivada es posible obtener una variación más pronunciada producto del cálculo de diferencias obtenido.

$$
y^{\prime \prime \prime}\left(x_{k}\right)=\frac{y\left(x_{k+2}\right)-3 * y\left(x_{k+1}\right)+3 * y\left(x_{k-1}\right)-y\left(x_{k-2}\right)}{T^{3}}
$$

BIOTECH \& ENGINEERING Untels. Jul - Dic.1(2), 2021; ISSN:2788 -4295; 41-51 


\section{Resultados}

Las señales adquiridas son muestreadas a una frecuencia de muestreo de $5 \mathrm{kHz}$.

La figura 8 y figura 9 muestran los resultados obtenidos para el calculo de la 3ra derivada con la ecuación 4 de la corriente sobre una carga resistiva en operación normal (figura 8) y la figura 9 muestra la corriente y la respuesta de la tercera derivada para una carga resistiva con presencia de fallas de arco.

La figura 10 muestra el resultado obtenido para el cálculo de la tercera derivada para una carga de tipo regulador de intensidad en operación normal y con presencia de una falla de tipo arco eléctrico serie. En esta figura podemos observar que las señales del funcionamiento normal y en presencia de un arco eléctrico son muy similares, y por ende la detención del calculo de la tercera derivada presenta una respuesta muy similar para ambos casos.

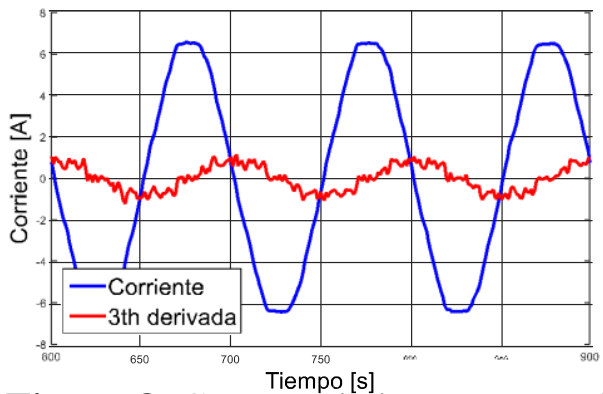

Figura 8. Carga resistiva en operación normal

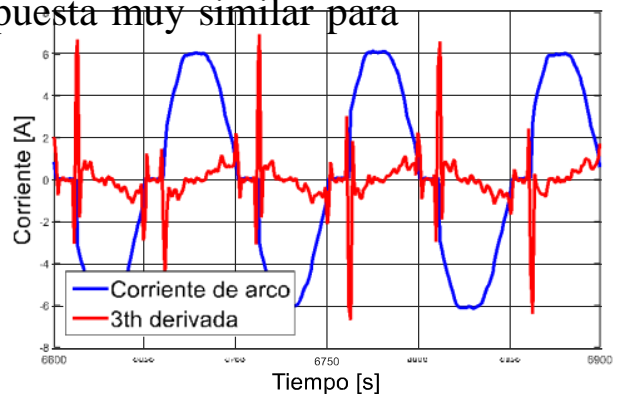

Figura 9. $3^{\text {ra }}$ derivada de corriente con falla de arco 


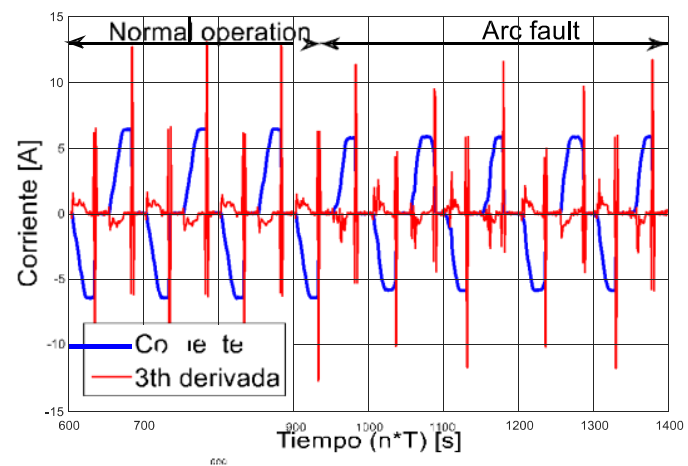

Figura 10. 3ra Derivada de corriente para una carga de tipo regulador de intensidad.

De acuerdo al análisis realizado, un arco eléctrico serie es un fenómeno que presenta una característica fuertemente caótica, no repetitiva (Tisserand, 2015), el cual es ligado a condiciones de aparición de una descarga influenciada por la naturaleza de los electrodos, la temperatura, la presión, la composición del aislante (gas, cables, etc.) y por el tipo de carga. Es de acuerdo a esta observación que se plantea la ecuación 5, la cual evidencia la diferencia existente entre el cálculo de la 3ra derivada entre 2 periodos consecutivos.

$$
\text { Diff } f_{3 e D}=\left[C_{3 e D}(i)-C_{3 e D}(i-N)\right]
$$

Donde: $\mathrm{C} 3 \mathrm{eD}$ son los valores del cálculo de la 3ra derivada, y $\mathrm{N}$ es el número de muestras por periodo. De acuerdo a la aplicación de la ecuación 5, la figura 11 muestra los resultados obtenidos para una señal del variador de intensidad en operación normal y con la presencia de una falla de arco eléctrico.

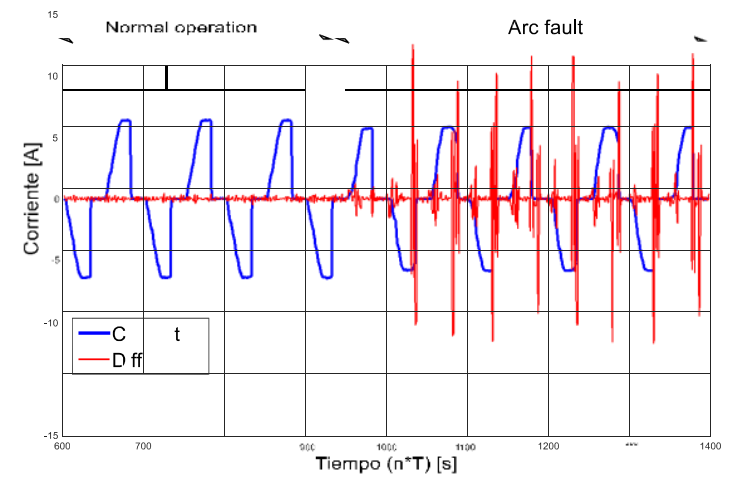

Figura 11. Calculo de la diferencia (Diff3eD) de la 3ra derivada para una carga de tipo variador de intensidad. 
La figura 12 muestra el resultado obtenido de la aplicación del método basado en la ecuación 5 para una carga resistiva en operación normal y con presencia de falla de arco eléctrico serie.

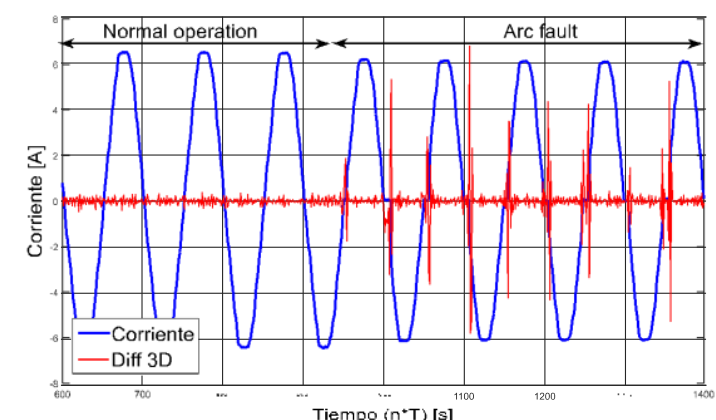

Figura 12. Diff3eD para una carga de tipo resistivo

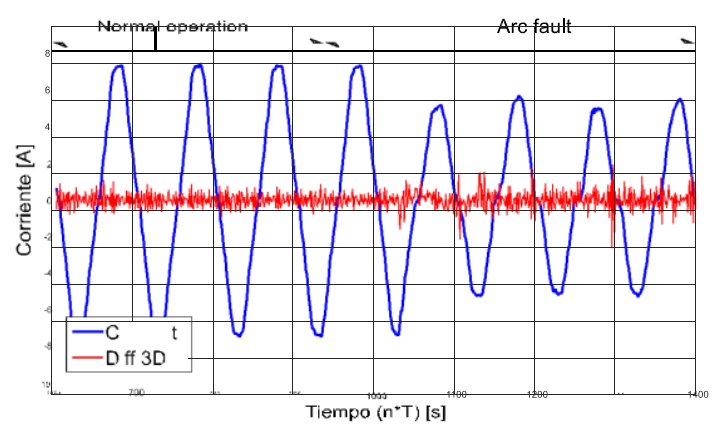

Figura 13. Diff3eD para una carga de tipo motor

La figura 13 muestra el resultado obtenido para una carga de tipo aspirador en operación normal y con presencia de falla de arco eléctrico serie.

La figura 14 muestra el resultado obtenido de la aplicación del método basado en la ecuación 5 para una carga de tipo variador de intensidad en operación normal y con presencia de falla de arco eléctrico serie. La figura 15 muestran los resultados obtenidos para una carga escondida o en combinación de carga (comprendía en una carga resistiva en paralelo con el variador de intensidad y la falla de arco eléctrico serie). 


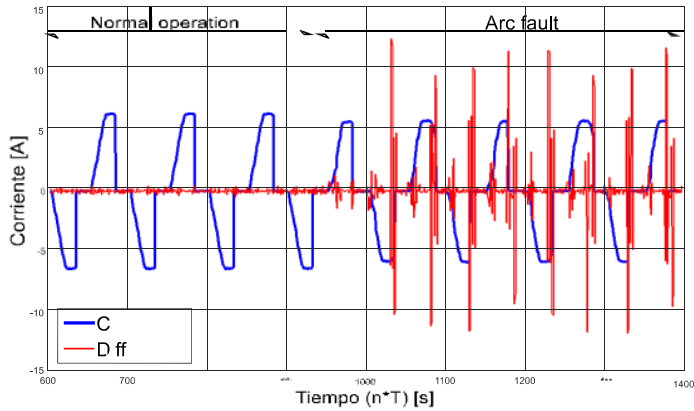

Figura 14. Diff3eD para una carga de tipo variador de intensidad.

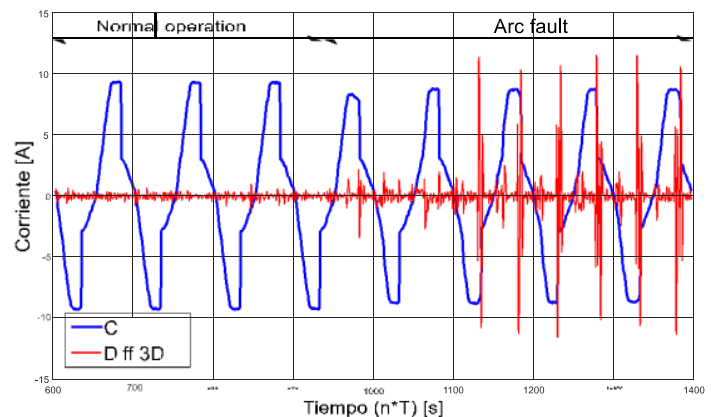

Figura 15. Diff3eD para una carga de tipo escondida.

Pese a los buenos resultados observados, el método presentado es aún sensible al ruido y variaciones relativas a los fenómenos de la línea eléctrica. Siendo de esta forma que los resultados pueden conducir a falsas detecciones de fallas de arco, sobre todo e el caso de cargas de tipo motor o aspirador.

\section{Conclusiones}

El articulo presenta el trabajo realizado para la adquisición de señales de corriente (forma de onda) de distintas cargas y configuraciones. El método planteado ha permitido observa y aprovechar los cambios rápidos de corriente cuando una descarga (tensión de restrike) es producida. Este método es basado en la 3ra derivada, adicionalmente, se propone la mejora del método de detección, explotando una de las características importantes de las fallas de arco eléctrico, como lo es la no repetitividad del fenómeno entre dos periodos consecutivos. Los resultados han mostrado que es posible la detección para distintos tipos de falla de arco eléctrico, sin embargo, existen algunas combinaciones y cargas, como la de tipo motor, que dificulta su detección con este método. La siguiente etapa de este trabajo consistirá en la implementación de los algoritmos en un dispositivo embebido para su análisis en tiempo real. 


\section{Referencias Bibliográficas}

Underwriters Laboratories (2008). UL Standard for safety for Arc Fautl Circuit Interrupter. ANSI UL 1699, vol. 2, p. 122.

IEC62606 (2013). General requeriments for arc fault detection devices. International Electrotechnical Commission. First Edition, p. 150.

Floyd, H. L., Andrews, J. J., Capelli-Schellpfeffer, M. A. R. Y., \& Liggett, D. P. (2003). An overview of the state-of-the-art in electrical safety technology, work practices and management systems. In Conference Record of the 2003 Annual Pulp and Paper Industry Technical Conference, 2003. (pp. 123-140). IEEE.

Johnson, J., \& Kang, J. (2012, June). Arc-fault detector algorithm evaluation method utilizing prerecorded arcing signatures. In 2012 38th IEEE Photovoltaic Specialists Conference (pp. 001378-001382). IEEE.

Tisserand, E., Lezama, J., Schweitzer, P., \& Berviller, Y. (2015). Series arcing detection by algebraic derivative of the current. Electric Power Systems Research, 119, 9199.

Le, V., Yao, X., Miller, C., \& Tsao, B. H. (2020). Series dc arc fault detection based on ensemble machine learning. IEEE Transactions on Power Electronics, 35(8), 78267839 .

Artale, G., Cataliotti, A., Cosentino, V., Di Cara, D., Nuccio, S., \& Tine, G. (2017). Arc fault detection method based on CZT low-frequency harmonic current analysis. IEEE Transactions on Instrumentation and Measurement, 66(5), 888-896.

Yang, K., Chu, R., Zhang, R., Xiao, J., \& Tu, R. (2020). A novel methodology for series arc fault detection by temporal domain visualization and convolutional neural network. Sensors, 20(1), 162.

Bao, G., Gao, X., Jiang, R., \& Huang, K. (2019). A novel differential high-frequency current transformer sensor for series arc fault detection. Sensors, 19(17), 3649.

Qi, P., Jovanovic, S., Lezama, J., \& Schweitzer, P. (2017). Discrete wavelet transform optimal parameters estimation for arc fault detection in low-voltage residential power networks. Electric Power Systems Research, 143, 130-139.

Qu, N., Wang, J., \& Liu, J. (2018). An arc fault detection method based on current amplitude spectrum and sparse representation. IEEE Transactions on Instrumentation and Measurement, 68(10), 3785-3792. 